\title{
Editorial
}

\section{Violence can be prevented}

Violence permeates and profoundly affects almost every aspect of our lives in South Africa. It poses a significant threat to the overall health and well-being of our nation and has a negative impact on development. Major strides are being made internationally in how to best respond to provide services for survivors of violence, particularly in the field of gender-based violence. ${ }^{1}$ There is, however, an urgent need to complement what is being done in the field of response with primary prevention programming.

Violence can be prevented. Limited evidence on what works to prevent violence before it occurs and the capacity to develop and deliver evidence-based programmes have been major barriers to the prevention agenda - but this is changing. South Africa is leading the way in building evidence for the primary prevention of violence in low- and middle-income countries, particularly in the area of gender-based violence. Promising practices in stopping violence before it starts, based on clear theoretical frameworks, informed by local practices and rooted in risk factors that drive the violence, are available for adaptation and potential scale-up in South Africa. ${ }^{2}$ This special edition of SACQ is dedicated to the violence prevention efforts currently underway in the country.

Violence prevention generally falls into three categories: primary, secondary and tertiary. Simply put, primary prevention of violence includes programmes that aim to prevent violence before someone is harmed, while secondary and tertiary prevention are those programmes that intervene early, or follow after violence has occurred, aiming to prevent its recurrence. Primary prevention programmes usually engage with all people, whereas secondary and tertiary prevention programmes work with high-risk groups, victimsurvivors or perpetrators.

In this edition of $S A C Q$ we focus on primary prevention programmes - those programmes aiming to address the factors that increase the risk of someone perpetrating or becoming a victim of violence. It is important to highlight that all forms of prevention (primary, secondary and tertiary) are important, and in many instances are connected.

Understanding what drives violence is a critical aspect of primary prevention programmes. Violence against women, for example, is fundamentally about gender inequality, and is to a great extent related to gender norms and relationship factors. Men's use of violence is also related to beliefs about masculinity, i.e. being tough, sexual performance and being dominant over women. Men's experiences of violence, particularly in childhood, are strongly associated with their perpetration of violence against women as adults. ${ }^{3}$

An increasing body of literature shows us that there are linkages between different forms of violence. It is therefore not surprising that in some instances different forms of violence share the same risk factors. ${ }^{4}$ This research has implications for intervention and prevention programmes: firstly, several risk factors are rooted in childhood and thus violence prevention efforts must begin in childhood; and secondly, the linkages between different forms of violence suggest that targeting key shared risk factors may strengthen the impact of violence prevention programmes.

By intervening early we can profoundly influence the life trajectory of children and invest in a long-term and longlasting violence prevention effort. In their commentary, Sarah Skeen and colleagues note that early intervention sets a strong foundation for lifelong violence prevention. Compelling research by Cathy Ward and colleagues on the connections between parenting, childhood aggression and mental health shows how parenting stress, parents' relationship difficulties and their mental health problems have a direct impact on the behaviour of their 
children, thus concluding that interventions supporting parents are essential in violence prevention. Parenting programmes that help parents develop safe and secure attachments with their children, promote positive discipline strategies with their children, and implement mental health promotion and adaptive coping strategies in their lives can inoculate against the contagion of violence throughout lives and across generations. This is further supported by Joanne Phyfer and Lorenzo Wakefield. In their article they argue for increased intersections between the delivery of early childhood development services and the primary prevention of violence in South Africa, but note the lack of a policy framework for the nationwide scale-up of evidence-based programmes.

Evidence-based programmes are those programmes that are well designed; thoughtful; build on what has been done before and has been found to be effective; informed by a theoretical model (risk/protective factors); guided by formative research and successful pilots; and are multi-faceted and address several causal factors. South Africa is home to some of the few sexual and intimate partner violence prevention programmes that have been rigorously tested and found effective in a low resource setting. A summary of these is discussed in the article by Nwabisa Jama-Shai and Yandisa Sikweyiya. The challenge for us as a country is to scale up what we know to be effective.

Other programmes yet to be adapted and tested in South Africa, but worthy of our attention, include programmes from the African region such as the SASA! Programme, which engages with communities to change gender social norms, ${ }^{5}$ and Safe Homes and Respect for Everyone (SHARE), which aims to prevent intimate partner violence by transforming community attitudes about women's status and the acceptability of violence against women. ${ }^{6}$ Both these programmes have been rigorously evaluated and found impactful.

Policymakers must ensure that their violence prevention policies and programmes are evidence based. To do this, they need to understand what constitutes good evidence, be prepared to invest time and resources in rigorous evaluations of programmes, and work with researchers to keep abreast of the field, thus ensuring that public funds are spent on what is currently understood to be effective.

Mark Tomlinson and colleagues argue that evaluation of programmes should be an essential part of public investment. They provide guidance on what is viewed as best evidence and what type of evidence we should use to make decisions on where to invest precious public funds.

South Africans are at the forefront of the field of primary prevention of violence in low- and middle-income countries, with several effective or promising programmes for prevention being developed and tested in the country. More work and resources are needed to build capacity and scale up these evidenced-based programmes. We have the tools - now we need the political will, resources and strategies to take these programmes to scale to move toward a safe and vibrant South Africa for all.

\section{Elizabeth Dartnall and Anik Gevers (Guest editors)}

\section{Notes}

1 World Health Organization (WHO), Responding to intimate partner and sexual violence against women: WHO clinical and policy guidelines, DoRHa Research, Geneva: WHO, 2013.

$2 \mathrm{WHO}$ and the London School of Hygiene and Tropical Medicine, Preventing intimate partner and sexual violence against women: taking action and generating evidence, 2010.

$3 \mathrm{M}$ Contreras et al, Bridges to adulthood: understanding the lifelong influence of men's childhood experiences of violence, Washington DC: International Center for Research on Women and Rio de Janeiro: Instituto Promundo, 2012; R Jewkes et al, Prospective study of rape perpetration by young South African men: incidence \& risk factors for rape perpetration, PLoS ONE, 7:5, 2012, e38210; E Fulu et al, Why do some men use violence against women and how can we prevent it?, United Nations Multi-Country Study on Men and Violence in Asia and the Pacific, Quantative Findings, 2013.

$4 \quad$ N Wilkins et al, Connecting the dots: an overview of the links among multiple forms of violence, Atlanta: National Center for Injury Prevention and Control and Oakland: Prevention Institute, 2014.

5 T Abramsky et al, Findings from the SASA! study: a cluster randomized controlled trial to assess the impact of a community mobilization intervention to prevent violence against women and reduce HIV risk in Kampala, Uganda, BMC Med, 31;12, $2014,122$.

6 J Wagman et al, A public health approach to intimate partner violence prevention in rural Uganda: the SHARE Project, Violence Against Women, 18:12, 2012, 1390-412. 